\title{
Influence of smoking and alcohol on gastric chemokine mRNA expression in patients with Helicobacter pylori infection
}

\author{
T Shimoyama, S M Everett, S Fukuda, A T R Axon, M F Dixon, J E Crabtree
}

\begin{abstract}
Aim-Chemokines that play a primary role in active inflammation are increased in gastric mucosa infected with Helicobacter pylori. Cigarette smoking increases the risk of peptic ulcer disease and gastric cancer, whereas alcohol might exert an antibacterial role. The aim of this study was to examine the association between smoking or alcohol consumption and mucosal chemokine mRNA expression in $H$ pylori associated gastritis.

Methods-Gastric biopsy specimens were obtained from 46 patients with dyspepsia who were infected with $H$ pylori, and total RNA was extracted. Semiquantitative reverse transcription-polymerase chain reaction (RT-PCR) was performed to quantify the mRNA expression of three C-X-C chemokines (interleukin 8 (IL-8), growth related oncogene $\alpha(\mathrm{GRO} \alpha)$, epithelial neutrophil activating protein 78 (ENA78)) and two C-C chemokines (regulated on activation normal $T$ cell expressed and secreted (RANTES) and monocyte chemotactic protein 1 (MCP-1)).

Results-GRO $\alpha$ and ENA-78 mRNA expression was significantly increased $(\mathbf{p}<0.05)$ in 22 smokers compared with 24 non-smokers; however, no difference was seen in the expression of IL-8, RANTES, and MCP-1 mRNA. No differences were observed in chemokine mRNA expression in relation to alcohol consumption.

Conclusions-The increased C-X-C chemokine mRNA expression seen in smokers might play a role in inducing enhanced inflammatory activity in gastritis and the consequent severe diseases associated with $\boldsymbol{H}$ pylori infection.

(F Clin Pathol 2001;54:332-334)
\end{abstract}

Keywords: Helicobacter pylori; C-X-C chemokine; smoking; alcohol

The chemokines are a family of inflammatory cytokines with leucocyte chemotactic and activating properties, which play a major role in regulating leucocyte populations migrating

Centre for Digestive Disease, Leeds General Infirmary, Leeds, UK A T R Axon

Correspondence to: Dr Shimoyama

tsimo-hki@umin.ac.jp

Accepted for publication 17 August 2000 into tissues. The chemokine superfamily has been divided into two major subgroups: the C-X-C and the C-C chemokines. ${ }^{1}$ C-X-C chemokines, which primarily affect neutrophils, have been detected in higher concentrations in gastric mucosa infected with Helicobacter pylori. ${ }^{2-4}$ Furthermore, semiquantitative analysis of C-X-C chemokine mRNA expression significantly correlates with the severity of gastritis. ${ }^{5}$ Infection with cagA positive $H$ pylori induces higher gastric $\mathrm{C}-\mathrm{X}-\mathrm{C}$ chemokine mRNA expression, which might be relevant to the increased mucosal damage associated with cagA positive strains. ${ }^{5}$

The possible effects of lifestyle factors have been studied in upper gastrointestinal diseases. Smoking is thought to affect gastric mucosa adversely by several mechanisms, ${ }^{6}$ and is a well established risk factor for peptic ulcer disease and gastric cancer. ${ }^{6}{ }^{7}$ On the other hand, recent studies have suggested a protective effect of alcohol consumption in $H$ pylori infection. ${ }^{8}$ In the lungs, smoking has been linked to raised cellular cytokines, ${ }^{9}$ but the effects of lifestyle factors on gastric mucosal chemokine mRNA expression in $H$ pylori infection have not been studied.

The aim of our study was to investigate whether smoking and alcohol consumption influence C-X-C chemokine mRNA expression in $H$ pylori infected gastric mucosa. We studied three C-X-C chemokines: interleukin 8 (IL-8), growth related oncogene $\alpha(\mathrm{GRO} \alpha)$, epithelial neutrophil activating protein 78 (ENA-78) and two C-C chemokines: regulated on activation normal $\mathrm{T}$ cell expressed and secreted (RANTES) and monocyte chemotactic protein 1 (MCP-1) using semiquantitative reverse transcription-polymerase chain reaction (RT-PCR).

Materials and methods

PATIENTS

Patients with dyspeptic symptoms who were to undergo upper gastrointestinal endoscopy at Leeds General Infirmary were enrolled prospectively into the study. Patients were excluded if they were taking steroids or nonsteroidal anti-inflammatory drugs or had received anti-ulcer agents or antibiotics during the two months before endoscopy. Infection with $H$ pylori was confirmed by a rapid biopsy urease test and histology. A total of 83 patients underwent endoscopy. Forty six patients were infected with $H$ pylori and hence eligible for inclusion. There were 23 men and 23 women and the mean age was 49.4 years (table 1 ). 
Table 2 Ratios of chemokine to glyceraldehyde 3-phosphate dehydrogenase (G3PDH) $m R N A$ expression in the antral mucosa

\begin{tabular}{llllll}
\hline & IL-8 & GROa & ENA-78 & \multirow{2}{*}{ RANTES } & MCP-1 \\
\hline Smoker & $1.15(0.16)$ & $1.45(0.12)^{\star}$ & $1.07(0.12)^{\star}$ & $0.48(0.12)$ & $0.74(0.15)$ \\
Non-smoker & $1.00(0.15)$ & $1.05(0.14)$ & $0.62(0.17)$ & $0.44(0.08)$ & $0.71(0.10)$ \\
Drinker & $1.08(0.16)$ & $1.23(0.13)$ & $0.79(0.14)$ & $0.44(0.13)$ & $0.67(0.18)$ \\
Non-drinker & $1.07(0.14)$ & $1.27(0.12)$ & $0.87(0.18)$ & $0.47(0.08)$ & $0.55(0.10)$ \\
\hline
\end{tabular}

Values are expressed as mean (SEM).

$\star$ Differences are significant between smokers and non-smokers $(p<0.05)$.

ENA-78, epithelial neutrophil activating protein 78 ; GRO $\alpha$, growth related oncogene $\alpha$; IL-8, interleukin 8; MCP-1, monocyte chemotactic protein 1; RANTES, regulated on activation normal $\mathrm{T}$ cell expressed and secreted.

Smoking habit and weekly alcohol intake were recorded before endoscopy. Of the 46 patients, 22 patients were smokers and 14 patients regularly consumed alcohol (patients who drank occasionally-less than 2 units/week-were included in the non-drinker group). Among smokers, the average number of cigarettes consumed each day was 12.0. The average number of units of alcohol drunk each week by regular consumers was 10.7 units (1 unit equals $12 \mathrm{~g}$ of alcohol). With respect to age, sex, and cagA positivity, there was no significant difference between frequency of smoking and alcohol consumption.

The histological features were graded as described in the updated Sydney system by one pathologist (MFD). ${ }^{10}$ In addition, polymorphonuclear (PMN) cell infiltration was graded using a visual analogue scale with units of 0 to 100. Two biopsy specimens obtained from the antrum were snap frozen and stored at $-80^{\circ} \mathrm{C}$ until use. All subjects provided informed consent before their endoscopy. Our study was approved by the local clinical research ethics committee (Leeds General Infirmary).

SEMIQUANTITATIVE RT-PCR

Total RNA was extracted from the frozen biopsy specimens using Catrimox-14 ${ }^{\mathrm{TM}}$ (Iowa Biotechnology Corp, Iowa, USA). Each RNA sample was suspended in water containing $1 \mathrm{U} / \mu \mathrm{l}$ of $\mathrm{RNasin}^{\mathrm{TM}}$ (ribonuclease inhibitor; Promega, Southampton, UK). A $10 \mu \mathrm{l}$ aliquot of each RNA solution was reverse transcribed using Random Primers ${ }^{\mathrm{TM}}$ (random hexamer; Promega). A $1 \mu$ l volume of the cDNA solution was added to $20 \mu \mathrm{l}$ reaction mixture and 30 cycles (chemokines and glyceraldehyde 3-phosphate dehydrogenase (G3PDH; a house keeping gene)) or 40 cycles (cagA) of PCR were performed as described previously. ${ }^{5} \mathrm{PCR}$ products were electrophoresed in a $2 \%$ agarose gel. Quantities of chemokine PCR products were compared by densitometry, with G3PDH products as an internal control. The ratios of chemokine to G3PDH were calculated to express chemokine mRNA expression semiquantitatively. ${ }^{5}$

\section{STATISTICAL ANALYSIS}

Results of chemokine to G3PDH ratios were expressed as mean (SEM) and were compared by the two tailed $t$ test. The degree of PMN infiltration was expressed as mean (SD) and results were also compared by the two tailed $t$ test. Differences between characteristics of patient groups were compared by the two tailed $t$ test or $\chi^{2}$ test. Determinations of Pearson's correlation coefficient were also made to examine the relation between the quantity of cigarettes and the scores of PMN infiltration or chemokine mRNA expression. A p value $<0.05$ was considered to be significant.

\section{Results}

Table 2 summarises the ratios of chemokine to G3PDH mRNA expression. GRO $\alpha$ and ENA-78 mRNA expression was significantly higher in smokers than in non-smokers $(\mathrm{p}<0.05)$, whereas no difference was seen in IL-8, RANTES, and MCP-1 mRNA expression. The mean (SEM) ratio of GRO $\alpha$ to G3PDH was $1.45(0.12)$ in patients who smoked and $1.05(0.14)$ in non-smoking patients $(p<0.05)$. The mean $($ SEM) ratio of ENA-78 to G3PDH was 1.07 (0.12) and 0.62 $(0.17)$ in smokers and non-smokers, respectively $(\mathrm{p}<0.05)$.

Chemokine mRNA expression was also compared in patients with cagA positive infection. In the 27 cagA positive patients, mean (SEM) expression of ENA-78 mRNA in smokers was $1.38(0.20)(n=14)$, which was significantly higher than that of non-smokers (0.77 (0.18); $\mathrm{p}<0.05 ; \mathrm{n}=13)$. Mean (SEM) expression of IL-8 and GRO $\alpha$ mRNA was 1.52 (0.10) and $1.64(0.16)$, respectively, in smokers with cagA positive infection. These values were higher than those seen in cagA positive non-smokers (mean (SEM) 1.45 (0.18) and 1.40 (0.16), respectively) but the differences were not significant. There was no significant correlation between the numbers of cigarettes and chemokine mRNA expression. The calculated correlation coefficient were $0.094(95 \%$ confidence interval (CI), -0.341 to 0.496 ) for IL-8, 0.065 (95\% CI, -0.367 to 0.473 ) for GRO $\alpha$, and 0.065 (95\% CI, -0.441 to 0.468$)$ for ENA-78.

The expression of both RANTES and MCP-1 mRNA was similar in smokers with cagA positive or negative infection. No difference in chemokine mRNA expression was seen in the 14 patients who consumed alcohol and the 32 with no alcohol consumption (table 2).

The degree of PMN infiltration was higher in smokers: mean (SD) 34.3 (18.9) compared with 23.6 (16.9) for non-smokers ( $p<0.05)$. The calculated correlation coefficient between the quantity of cigarettes and PMN infiltration was 0.134 (95\% CI, -0.304 to 0.526 ; not significant). Alcohol consumption was not associated with PMN infiltration. The mean (SD) degree of PMN infiltration was 29.0 (20.6) in alcohol consumers and $28.6(17.7)$ in the nonconsumers.

\section{Discussion}

It is well established that smoking is associated with an increased risk of peptic ulcer disease and gastric cancer. ${ }^{7}$ Several studies have investigated the mechanisms by which smoking might induce gastric mucosal injury. The potentiating effects of smoking on gastritis caused by $H$ pylori infection are thought to relate to the increased production of oxygen derived free radicals and a decrease in gastric mucus content. ${ }^{68}$ Active $H$ pylori gastritis is 
characterised histologically by an infiltration of PMNs, ${ }^{10}$ which are a major source of oxygen derived free radicals. ${ }^{11}$ In our previous study, the expression of mRNA encoding the C-X-C chemokines, GRO $\alpha$ and ENA-78, positively correlated with PMN infiltration in $H$ pylori positive gastric mucosa. ${ }^{5}$ The increased expression of C-X-C chemokine mRNA in smokers could account for the increased PMN infiltration in their gastric mucosa. Infection with cagA positive $H$ pylori is strongly associated with C-X-C chemokine expression. ${ }^{5}$ In our study, however, within the group of cagA positive infected patients studied, smoking was also associated with increased ENA-78 mRNA expression but the increase of expression of other C-X-C chemokine mRNA was not significant. The limited sample size might preclude us from finding a significant difference in the expression of IL- 8 or GRO $\alpha$ mRNA.

The potentiation of $H$ pylori cytotoxin activity by nicotine, ${ }^{12}$ which is an important toxic component of smoking, might indirectly play a role in the induction of $\mathrm{C}-\mathrm{X}-\mathrm{C}$ chemokine expression. VacA has no direct effect on the induction of epithelial chemokines by $H p y$ lori ${ }^{13}{ }^{14}$ but it might promote epithelial damage, uptake of bacterial components, and thus stimulation of macrophages ${ }^{15}$ and neutrophil chemokine production. ${ }^{16}$ Furthermore, C-X-C chemokines can also activate neutrophils to produce reactive oxygen metabolites. ${ }^{17}$ In smokers, the production of oxygen derived free radicals by peripheral PMNs is higher than in non-smokers. ${ }^{18}{ }^{19}$ In addition, smoking is known to inhibit the synthesis of gastric mucus and reduce plasma vitamin $\mathrm{C}$ concentrations, both of which are effective scavengers of oxidants produced in the gastric mucosa. ${ }^{20}$ These data suggest that oxygen derived free radicals might play a role in both gastric mucosal injury and oxidative DNA damage of gastric epithelial cells in smokers infected with $H$ pylori.

Several studies have investigated the effects of alcohol on $H$ pylori infection. A recent study suggested a protective effect of alcohol against active $H$ pylori infection. ${ }^{8}$ This effect might relate to the antimicrobial effects of alcohol. ${ }^{21}$ In our present study, gastric mucosal C-X-C chemokine mRNA expression did not differ between those who did or did not consume alcohol, despite the fact that 10 of the 14 drinkers were smokers. Although these results might suggest that alcohol consumption decreases C-X-C chemokine expression, the number of patients was insufficient for further subgroup analysis.

In conclusion, we have demonstrated an association between smoking and raised gastric C-X-C chemokine expression in $H$ pylori associated gastritis. Increased chemokines might exacerbate the severity of gastritis and affect the disease outcome in smokers infected with $H$ pylori.
However, other potential confounding factors, such as dietary antioxidant consumption, should be studied to elucidate the effects of lifestyle on $H$ pylori associated gastritis.

These studies were undertaken with financial support from Yorkshire Cancer Research and the European Commission (contract number ICA4-CT-1999-10010). We thank Dr I Lindley of Novartis for providing GRO $\alpha$ primers and Dr S Farmery for helpful discussion. The authors thank Professor A Munakata and Dr S Nakaji for their useful discussion.

1 Luster AD. Mechanisms of disease: chemokineschemotactic cytokines that mediate inflammation. N Engl f Med 1998:338:436-45.

2 Crabtree JE, Peichl P, Wyatt JI, et al. Gastric IL-8 and IL-8 IgA autoantibodies in Helicobacter pylori infection. Scand fImmunol 1993:37:65-70.

3 Peek RM, Miller GG, Tham KT, et al. Heightened inflammatory response and cytokine expression in vivo to CagA+ Helicobacter pylori strains. Lab Invest 1995:73: 760-70.

4 Ando T, Kusugami K, Ohsuga M, et al. Interleukin-8 activity correlates with histological severity in Helicobacter pylori-associated antral gastritis. Am f Gastroenterol 1996: 91:1150-6.

5 Shimoyama T, Everett SM, Dixon MF, et al. Chemokine mRNA expression in gastric mucosa is associated with Helicobacter pylori cagA positivity and severity of gastritis. 7 Clin Pathol 1998;51:765-70.

6 Endoh K, Leung FW. Effects of smoking and nicotine on the gastric mucosa: a review of clinical and experimental evidence. Gastroenterology 1994:107:864-78.

7 Komoto K, Haruma K, Kamada T, et al. Helicobacter pylori infection and gastric neoplasia: correlations with histological gastritis and tumor histology. Am $f$ Gastroenterol 1998;93:1271-6.

8 Brenner H, Rothenbacher D, Bode G, et al. Relation of smoking and alcohol and coffee consumption to active Helicobacter pylori infection. BMF 1997:315:1489-92.

9 Morrison D, Strieter RM, Donnelly SC, et al. Neutrophil chemokines in bronchoalveolar lavage fluid and leukocyteconditioned medium from nonsmokers and smokers. Eur Respir F 1998;12:1067-72.

10 Dixon MF, Genta RM, Yardley JH, et al. Classification and grading of gastritis. The updated Sydney system. Am $\mathcal{F}$ Surg Pathol 1996;20:1161-81.

11 Jones-Blackett S, Hull MA, Davies GR, et al. Non-steroidal anti-inflammatory drugs inhibit Helicobacter pylorianti-inflammatory drugs inhibit Helicobacter pylorivitro. Aliment Pharmacol Ther 1999;13:1653-61.

12 Cover TL, Vaughn SG, Cao P, et al. Potentiation of Helicobacter pylori vacuolating toxin activity by nicotine and other weak bases. F Infect Dis 1992;166:1073-8.

13 Crabtree JE, Covacci A, Farmery SM, et al. Helicobacter pylori induced interleukin-8 expression in gastric epithelial cells is associated with CagA positive phenotype. $f$ Clin Pathol 1995:48:41-5.

14 Sharma SA, Tummuru MKR, Miller GG, et al. Interleukin-8 response of gastric epithelial cell lines to Helicobacter pylori stimulation in vitro. Infect Immun 1995: 63:1681-7.

15 Bliss CM, Golenbock DT, Keates S, et al. Helicobacter pylori lipopolysaccharide binds to CD14 and stimulates release of interleukin-8, epithelial neutrophil-activating peptide 78 , and monocyte chemotactic protein 1 by human monocytes. Infect Immun 1998:66:5357-63.

16 Kim JS, Jung HC, Kim JM, et al. Interleukin-8 expression by human neutrophils activated by Helicobacter pylori soluble proteins. Scand 7 Gastroenterol 1998:33:1249-55.

17 Furie MB, Randolph GJ. Chemokines and tissue injury. Am f Pathol 1995;146:1287-301.

18 Kalra J, Chaudhary AK, Prasad K. Increased production of oxygen free radicals in cigarette smokers. Int f Exp Pathol 1991;72:1-7.

19 Phull PS, Price AB, Thorniley MS, et al. Plasma free radical activity and antioxidant vitamin levels in dyspeptic patients: correlation with smoking and Helicobacter pylori infection. Eur 7 Gastroenterol Hepatol 1998;10:573-8.

20 Preston A. Cigarette smoking-nutritional implications. Prog Food Nutr Sci 1991;15:183-217.

21 Weisse ME, Eberly B, Person DA. Wine as digestive aid: comparative antimicrobial effects of bismuth salicylate and red and white wine. BMf 1995;311:1657-60. 